\title{
Class of solitary wave solutions of the one-dimensional Gross-Pitaevskii equation
}

\author{
Rajneesh Atre, ${ }^{1,2, *}$ Prasanta K. Panigrahi, ${ }^{1, \dagger}$ and G. S. Agarwal ${ }^{1,3, \ddagger}$ \\ ${ }^{1}$ Physical Research Laboratory, Navrangpura, Ahmedabad 380 009, India \\ ${ }^{2}$ School of Physics, University of Hyderabad, Hyderabad 500 046, India \\ ${ }^{3}$ Department of Physics, Oklahoma State University, Stillwater, Oklahoma 74078, USA \\ (Received 11 July 2005; revised manuscript received 7 December 2005; published 19 May 2006)
}

\begin{abstract}
We present a large family of exact solitary wave solutions of the one-dimensional Gross-Pitaevskii equation, with time-varying scattering length and gain or loss, in both expulsive and regular parabolic confinement regimes. The consistency condition governing the soliton profiles is shown to map onto a linear Schrödinger eigenvalue problem, thereby enabling one to find analytically the effect of a wide variety of temporal variations in the control parameters, which are experimentally realizable. Corresponding to each solvable quantum mechanical system, one can identify a soliton configuration. These include soliton trains in close analogy to experimental observations of Strecker et al. [Nature (London) 417, 150 (2002)], spatiotemporal dynamics, solitons undergoing rapid amplification, collapse and revival of condensates, and analytical expression of two-soliton bound states, to name a few.
\end{abstract}

DOI: 10.1103/PhysRevE.73.056611

PACS number(s): 03.75.Lm, 05.45.Yv, 03.75.-b

Coherent atom optics is the subject of much current interest due to its relevance to fundamental aspects of physics, as well as to technology [1-6]. For that purpose, lower dimensional condensates, e.g., cigar-shaped Bose-Einstein condensates (BECs) have been the subject of active study in the last few years [7-11]. Observations of dark and bright solitons [12-15], particularly the latter, since it is a condensate itself, have generated considerable interest in this area. This has spurred intense investigations about the behavior of condensates in the presence of time-varying control parameters. These include nonlinearity, achievable through Feshbach resonance [16-18], gain or loss, and the oscillator frequency [19]. The fact that for a condensate in an oscillator potential, exact solutions of the Gross-Pitaevskii (GP) equation are not available makes it extremely difficult to examine the effects of time variation in the aforementioned parameters. In the context of pulse propagation in nonlinear optical fibers, a number of authors have recently investigated the effects of variable nonlinearity, dispersion, and gain or loss. Moores analyzed this problem for constant dispersion and nonlinearity and a distributed gain [20], whereas Kruglov et al. considered the same problem with all the parameters in a variable form [21]. Serkin et al., have derived the nonlinear Schrödinger equation (NLSE) with distributed coefficients as a compatibility condition of two first order equations, demonstrating the applicability of the inverse scattering transform method to this type of problem [22]. They write down the general equation relating the distributed coefficients with the solution parameters and obtain a number of exact solutions. Recently, exact solutions of a driven NLSE with distributed dispersion, nonlinearity, and gain, which exhibits pulse compression in a twin-core optical fiber, have also been obtained [23].

In this paper, we present a large family of exact solutions

\footnotetext{
*Electronic address: atre@prl.res.in

${ }^{\dagger}$ Electronic address: prasanta@prl.res.in

${ }^{\ddagger}$ Electronic address: girish.agarwal@okstate.edu
}

of the quasi-one-dimensional GP equation, which is the familiar NLSE [24], with time-varying scattering length and gain or loss, in the presence of an oscillator potential, which can be either expulsive or regular. It is shown that the consistency condition governing the soliton profiles identically maps on to the linear Schrödinger eigenvalue problem, thereby allowing one to solve analytically the GP equation for a wide variety of temporal variations in the control parameters. Corresponding to each solvable quantummechanical eigenvalue problem, one can identify a solitonlike profile. These solitons can be dark or bright and the oscillator frequencies can have a variety of temporal profiles, including linear, quadratic, exponential, and smooth steplike potentials and the kicked oscillator scenario. It is worth mentioning that, in the fiber optics literature, bright solitons refer to localized structures with light intensities higher than the background and dark solitons to the ones with lower intensities as compared to the background. Our solutions exhibit soliton trains, spatiotemporal dynamics of solitons, and the formation of two-soliton bound states, to name a few. Interestingly, it was found that some of these solitons can periodically exchange atoms with a background. Further, our analytical results are closely related to experiments $[13,14]$. Amplification of the atomic condensate and condensate compression are observed in the parameter domain that is amenable for experimental verification.

Analogous to the experimental observations of Strecker et al., we obtain bright soliton trains in the presence of harmonic confinement [14]. Some of the soliton solutions in the presence of regular harmonic confinement, with appropriately tailored gain or loss, exhibit collapse and revival phenomena, with an increase in amplitude. We treat the attractive regime rather exhaustively since the bright solitons are themselves condensates. The analytic expression for the dark soliton in the repulsive sector has been presented; detailed analysis of the same can be carried out in a straightforward manner.

We start with a zero-temperature BEC of atoms, confined in a cylindrical harmonic trap $V_{0}(x, y)=m \omega_{\perp}^{2}\left(x^{2}+y^{2}\right) / 2$, and 

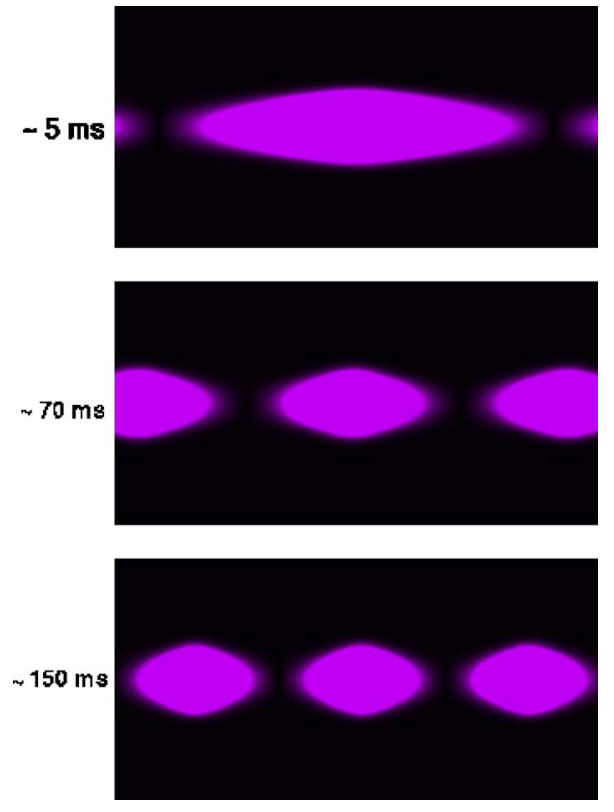

FIG. 1. (Color online) Snapshots of soliton trains at times $\approx 5$, $\approx 70$, and $\approx 150 \mathrm{~ms}$ with parameters $M=0.25, \gamma=-0.5, \tau_{0}=0.9999$, $A_{0}=0.5$, and modulus parameter $m=0.99999$.

a time-dependent harmonic confinement, which can be either attractive or expulsive, along the $z$ direction $V_{1}(z, t)$ $=m \omega_{0}^{2}(t) z^{2} / 2$ :

$$
i \hbar \frac{\partial \Psi(\mathbf{r}, t)}{\partial t}=\left(-\frac{\hbar^{2}}{2 m} \nabla^{2}+U|\Psi(\mathbf{r}, t)|^{2}+V+i \frac{\eta(t)}{2}\right) \Psi(\mathbf{r}, t),
$$

where $U=4 \pi \hbar^{2} a_{s}(t) / m$ and $V=V_{0}+V_{1}$. It is worth noting that the condensate can interact with the normal atomic cloud through three-body interaction, which can be phenomenologically incorporated by a gain or loss term $\eta(t)$.

To reduce Eq. (1) to an effective one-dimensional equation, we assume that the interaction energy of atoms is much less than the kinetic energy in the transverse direction [25]:

$$
\Psi(\mathbf{r}, t)=\frac{1}{\sqrt{2 \pi a_{B} a_{\perp}}} \psi\left(\frac{z}{a_{\perp}}, \omega_{\perp} t\right) \exp \left(-i \omega_{\perp} t-\frac{x^{2}+y^{2}}{2 a_{\perp}^{2}}\right) .
$$

In dimensionless units, the GP equation then reduces to the following one-dimensional nonlinear Schrödinger equation:

$$
i \partial_{t} \psi=-\frac{1}{2} \partial_{z z} \psi+\gamma(t)|\psi|^{2} \psi+\frac{1}{2} M(t) z^{2} \psi+i \frac{g(t)}{2} \psi .
$$

Here, $\quad \gamma=2 a_{s}(t) / a_{B}, \quad M(t)=\omega_{0}^{2}(t) / \omega_{\perp}^{2}, \quad g(t)=\eta(t) / \hbar \omega_{\perp}, \quad a_{\perp}$ $=\left(\hbar / m \omega_{\perp}\right)^{1 / 2}$, and $a_{B}$ is the Bohr radius. For the sake of generality we have kept $M(t)$ time dependent; a constant $M(t)$ implies an oscillator potential which can be confining or expulsive for $M>0$ or $M<0$, respectively.

In order to discuss the cases of a variety of experimentally achievable profiles of the time-dependent trapping potential and to obtain corresponding analytical solutions, we assume the following ansatz solution:

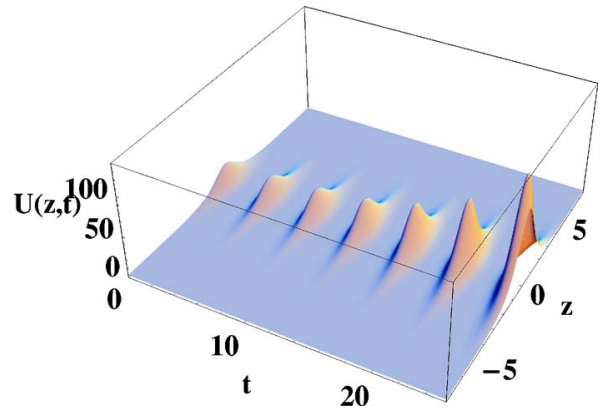

FIG. 2. (Color online) Collapse and revival of atomic condensate and amplification through periodic exchange of atoms with the background, for the parameter values $M=0.0025, \tau_{0}=2.5, A_{0}$ $=3.125, \gamma_{0}=-0.5$ as in the Ref. [14] and gain parameters $a_{1}=0.01$, $a_{2}=8.25$, and $\delta=1.5$.

$$
\psi(z, t)=\sqrt{A(t)} F\{A(t)[z-l(t)]\} \exp [i \Phi(z, t)+G(t) / 2],
$$

where $G(t)=\int_{0}^{t} g\left(t^{\prime}\right) d t^{\prime}, l(t)=\int_{0}^{t} v\left(t^{\prime}\right) d t^{\prime}$, and we assume that the phase has a quadratic form:

$$
\Phi(z, t)=a(t)+b(t) z-\frac{1}{2} c(t) z^{2} .
$$

In the above equation, $a(t)=a_{0}+\frac{\lambda-1}{2} \int_{0}^{t} A^{2}\left(t^{\prime}\right) d t^{\prime}$, and $c(t)$ is determined by a Riccati type equation:

$$
c_{t}-c^{2}(t)=M(t)
$$

Interestingly, this equation can be expressed as a Schrödinger eigenvalue problem via a change of variable, $c(t)=-\frac{d \ln [\varphi(t)]}{d t}$ :

$$
-\varphi^{\prime \prime}(t)-M(t) \varphi(t)=0
$$

Taking advantage of this connection, below we show that, corresponding to each solvable quantum-mechanical system, one can identify a soliton configuration. The fact that the Schrödinger equation can be exactly solved for a variety of $V(t)$ gives us freedom to control the dynamics of the BEC in a number of analytically tractable ways. This is one of the main results of this paper. Although a host of time-dependent oscillator frequencies can be addressed, we will only demonstrate in the text a few experimentally realizable examples.

We also find the following consistency conditions:

$$
\begin{gathered}
\gamma(t)=\gamma_{0} e^{-G(t) / 2} A(t) / A_{0}, \quad b(t)=A(t) \\
A(t)=A_{0} \exp \left\{\int_{0}^{t} c\left(t^{\prime}\right) d t^{\prime}\right\}, \quad A_{0}>0 \\
\frac{d l(t)}{d t}-c(t) l(t)=b(t)
\end{gathered}
$$

Now by substitution of the ansatz Eq. (4) in Eq. (3) and using the consistency conditions Eq. (8), we obtain the differential equation for the function $F$ in terms of the new variable $T=A(t)[z-l(t)]$ : 


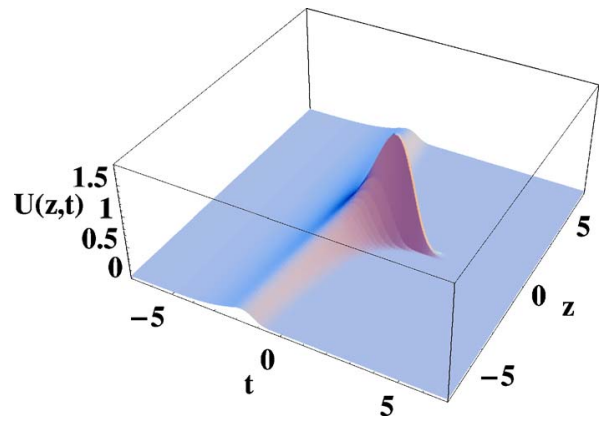

FIG. 3. (Color online) Formation of soliton in a trap, having a smooth steplike oscillator frequency profile.

$$
F^{\prime \prime}(T)-\lambda F(T)+2 \kappa F^{3}(T)=0 \quad \text { where } \kappa=-\frac{\gamma_{0}}{A_{0}} .
$$

We note that Eq. (9) possesses a variety of solutions in the form of 12 Jacobian elliptic functions, e.g., $\operatorname{cn}(T, m)$, $\operatorname{sn}(T, m)$, and $\operatorname{dn}(T, m)$, etc. Here $m$ is the modulus parameter taking value in the interval $0 \leqslant m \leqslant 1$. These functions interpolate between the trigonometric and hyperbolic functions in the limiting cases $m=0$ and 1, respectively. For example, $\operatorname{cn}(x, 1)=\operatorname{dn}(x, 1)=\operatorname{sech}(x)$ and $\operatorname{cn}(x, 0)=\cos (x)$, etc. Below, we give some useful identities satisfied by these functions, relevant for the calculations in this paper [26]:

$$
\begin{gathered}
\frac{d \operatorname{sn}(x, m)}{d x}=\operatorname{cn}(x, m) \operatorname{dn}(x, m), \\
\frac{d \operatorname{cn}(x, m)}{d x}=-\operatorname{sn}(x, m) \operatorname{dn}(x, m), \\
\frac{d \operatorname{dn}(x, m)}{d x}=-m \operatorname{cn}(x, m) \operatorname{sn}(x, m), \\
\operatorname{cn}^{2}(x, m)+\operatorname{sn}^{2}(x, m)=1, \\
m \operatorname{sn}^{2}(x, m)+\operatorname{dn}^{2}(x, m)=1 .
\end{gathered}
$$

In the appropriate parameter regimes, a wide variety of solutions emerge, which include soliton trains, akin to the experimental observations of Strecker et al., and localized

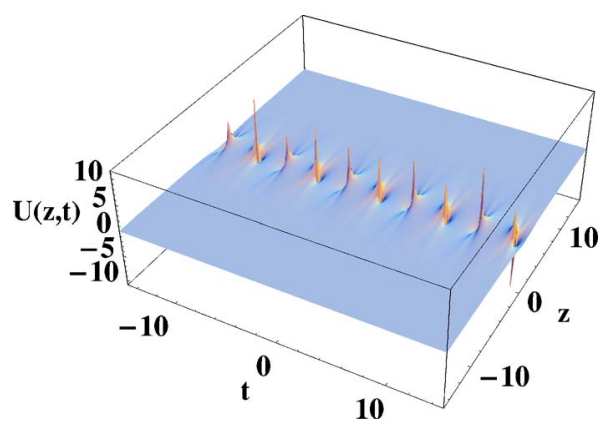

FIG. 4. (Color online) Spatiotemporal pattern of condensate wave function in a confining oscillator potential $[M(t)=0.25]$ with constant attractive coupling $\gamma(t)=-0.5$ and $A_{0}=0.5, \tau_{0}=1$.

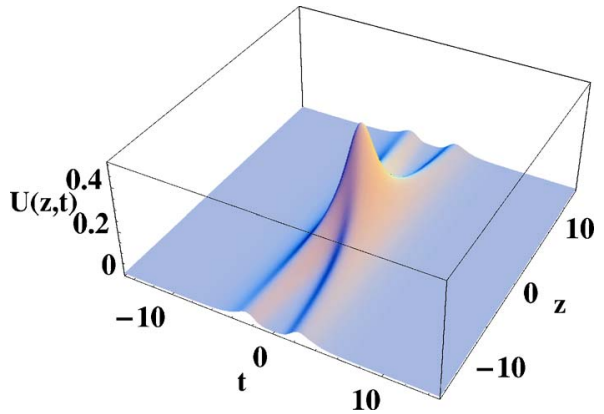

FIG. 5. (Color online) Formation of two-soliton bound state in an expulsive potential $[M(t)=-0.25]$ with all the other parameters having the same values as in Fig. 4.

bright and dark solitons. In a BEC, a bright soliton corresponds to density lumps and a dark soliton corresponds to density rarefactions.

Bright soliton trains of the form $\psi(z, t)$ $=\sqrt{A(t)} \operatorname{cn}\left(T / \tau_{0}, m\right) \exp [i \Phi(z, t)+G(t) / 2]$ exist for $\kappa>0$, where $\tau_{0}^{2}=-A_{0}\left(m+m^{2}\right) / 2 \gamma_{0}$ and $\lambda=\left(m^{2}+m-1\right) / 2 \tau_{0}^{2}$. Here, all the coefficients are determined from Eqs. (5)-(8). In the limiting case for $m=1$ it corresponds to a bright soliton of the type $\operatorname{sech}(T)$. It should be pointed out that in the repulsive domain $\gamma_{0}>0$ the localized dark soliton is of the form $\psi(z, t)=\sqrt{A(t)} \tanh \left[A(t) z / \tau_{0}\right] \exp [i \Phi(z, t)+G(t) / 2]$, since it vanishes at the origin. The cnoidal wave solution for this is of $\operatorname{sn}(T, m)$ type.

Below, we examine the formation and dynamics of nonlinear excitations in the presence of an oscillator potential, with a variety of experimentally achievable temporal modulations in the frequency.

Soliton trains in a confining oscillator. Inspired by the experiments of Strecker et al. [14], in the presence of a confining oscillator, with no gain or loss we obtain following moving soliton trains with velocity $v(t)=A_{0} \cos \left(M_{0} t\right)$ :

$$
\begin{aligned}
\psi(z, t)= & \sqrt{A_{0} \sec \left(M_{0} t\right)} \\
& \times \operatorname{cn}\left(\frac{A_{0} \sec \left(M_{0} t\right)\left[z-\frac{A_{0}}{M_{0}} \sin \left(M_{0} t\right)\right]}{\tau_{0}}, m\right) e^{i \Phi} .
\end{aligned}
$$

As shown in Fig. 1 initially at $\approx 5 \mathrm{~ms}$ there is only one profile and as time progresses it breaks up into many profiles giving rise to a soliton train.

Collapse and revival of the condensate. Inspired by the experiments of Strecker et al., we tailor the gain profile in the same range of the parameters where one observes dramatic collapse and revival of condensates with an increase in the amplitude. Specifically, we take $M(t)=M_{0}^{2}, g(t)=a_{1} t$ $-a_{2} \sin (\delta t)$, and $\gamma(t)=\gamma_{0} \exp \left[\frac{-a_{1} t^{2}}{2}-\frac{a_{2}}{\delta} \cos (\delta t)\right] \sec \left(M_{0} t\right)$, for which we obtain from Eq. (8), $A(t)=A_{0} \sec \left(M_{0} t\right)$. The complete solution can be written as 


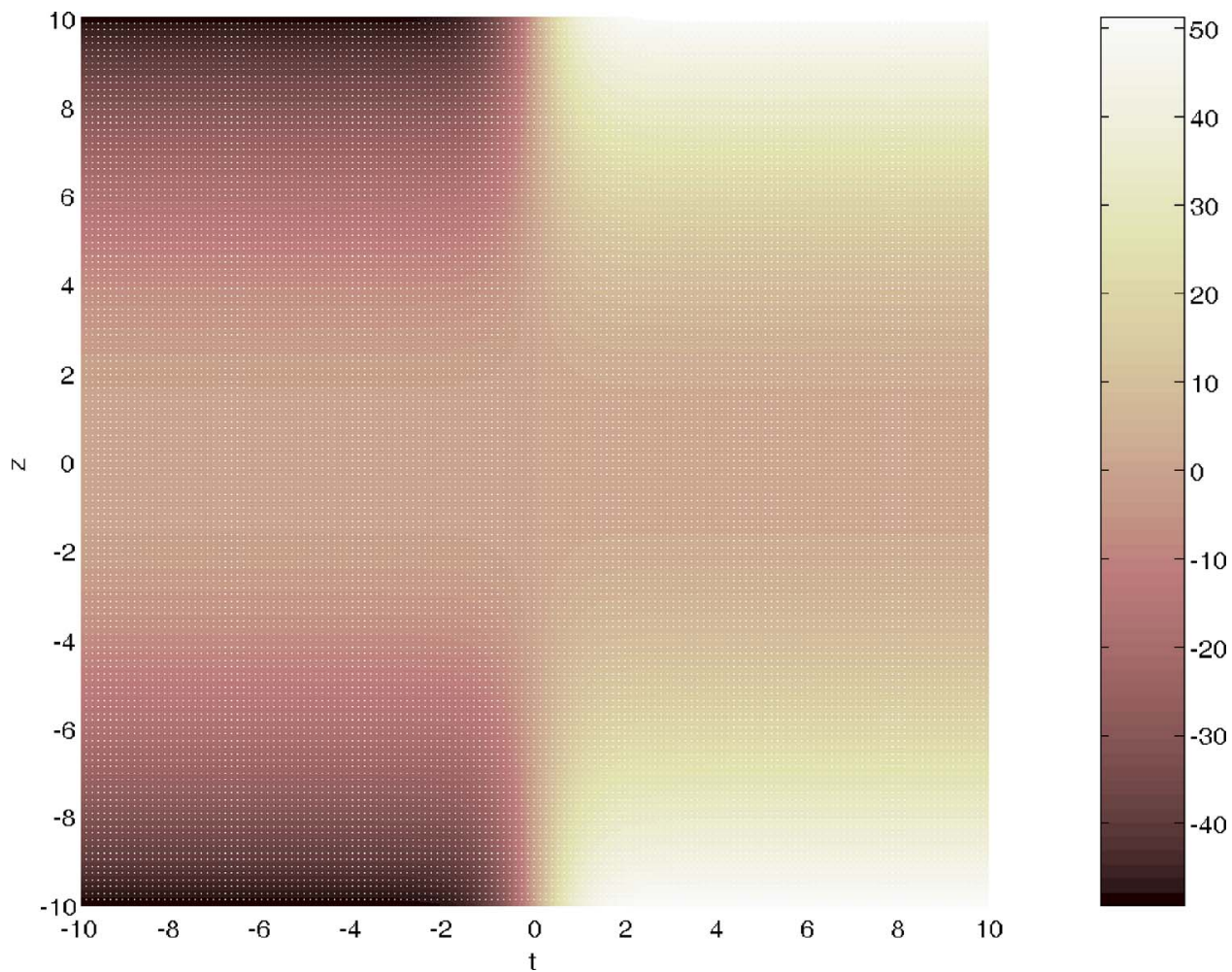

FIG. 6. (Color online) Variation of phase $\Phi(z, t)$, for the soliton bound state, with parameter values the same as in Fig. 5.

$$
\psi(z, t)=\sqrt{A_{0} \sec \left(M_{0} t\right)} \operatorname{sech}\left(\frac{A_{0} \sec \left(M_{0} t\right) z}{\tau_{0}}\right) \exp [G(t) / 2] e^{i \Phi}
$$

This is depicted in Fig. 2. In this case, sinusoidal nature of gain function implies a periodic exchange of atoms with the background. In the attractive domain, presence of a background surrounding the condensate has been seen in experiments, leading to formation of bright solitons $[13,14]$. In this light, the collapse and revival of the condensate, having a sinusoidal exchange of atoms with the background, may be amenable for verification.

Soliton formation from step changes in the trap potential. Below, we explicate in the attractive domain $\left(\gamma_{0}<0\right)$ the effect of a steplike change in the oscillator frequency which is well mimicked by the function $M(t)=-4+\frac{3}{2}[1$ $+\tanh (t / 2)]$, for which the coupling has the form $\gamma(t)$ $=\gamma_{0}\left[\frac{e^{2 t}}{\left(1+e^{t}\right)}\right], \gamma_{0}<0$. As time progresses, the coupling strength changes exponentially and the condensate wave function starts building up rapidly, as depicted in Fig. 3. In this expulsive case, as for the experimentally observed bright soliton [13], one obtains

$$
\psi(z, t)=\sqrt{\frac{A_{0} e^{2 t}}{\left(1+e^{t}\right)}} \operatorname{sech}\left(\frac{A_{0} e^{2 t} z}{\left(1+e^{t}\right) \tau_{0}}\right) \exp [i \Phi(z, t)] .
$$

Additional solutions in the case of constant coupling. A number of interesting condensate profiles emerge in the constant attractive coupling regime, depending on the nature of the external potential. For the regular oscillator confinement one obtains a spectacular spatiotemporal pattern given by Eq. (17) in the amplitude of the order parameter, as seen in
Fig. 4. The extreme increase in amplitude happens due to the presence of the $\sec \left(M_{0} t\right)$ in the amplitude:

$$
\psi(z, t)=\sqrt{A_{0} \sec \left(M_{0} t\right)} \operatorname{sech}\left(\frac{A_{0} \sec \left(M_{0} t\right) z}{\tau_{0}}\right) \exp (G / 2) e^{i \Phi}
$$

Additional bound state for solitons. Interestingly, as shown in Fig. 5, in the expulsive domain one obtains bound states of solitons in the same parameter regime. The analytical expression for this remains the same as in Eq. (17), except for the value of $A(t)$ which is now given by $A(t)$ $=A_{0} \operatorname{sech}\left(M_{0} t\right)$. It is worth mentioning that, in optical fibers with a variable dispersion, similar structures have been seen through numerical investigations [27].

Keeping in mind the nontrivial nature of the phase and its connection with the soliton profile, the nature of the spatiotemporal dynamics of the same for various configurations is worth discussing. The quadratic nature of the phase with respect to the space coordinate introduces a spatial chirping effect. Furthermore, its coefficient $c(t)$ is also time dependent and is connected with the external potential through Eq. (6); hence the trap potential has a signature in the coefficient. The purely temporal part $a(t)$ is also sensitive to the trap potential as depicted by Eq. (8). The case of a soliton bound state, where the phase varies smoothly across the time boundary for small $z$ values and rather abruptly for larger values of $z$, is depicted in Fig. 6.

In conclusion, for the one-dimensional GP equation in an oscillator potential, with time-dependent coupling and gain or loss, we have obtained a wide class of exact solutions. These include the experimentally observed bright soliton 
profile in the attractive coupling regime for the expulsive case. The fact that the equation governing the soliton profiles in the presence of a time-dependent harmonic oscillator maps to the Schrödinger equation opens up a host of opportunities. Corresponding to any solvable quantum-mechanical potential, a time-dependent cigar-shaped BEC profile can be obtained. We have explicated the amplification of the BEC profile through smooth variation of the oscillator frequency.
Formation of soliton bound states and spectacular spatiotemporal patterns that can manifest in this nonlinear system with time-dependent control parameters are demonstrated. One observes dramatic compression and localization of broad condensate profiles, which may have technological implications. We have analyzed the nonlinear excitations in the presence of oscillator potentials having linear, quadratic, Morse, and other types of time dependencies.
[1] G. Lenz, P. Meystre, and E. M. Wright, Phys. Rev. Lett. 71, 3271 (1993).

[2] Y. J. Wang et al., Phys. Rev. Lett. 94, 090405 (2005); Y. Shin et al., e-print cond-mat/0506464.

[3] Th. Busch et al., Phys. Rev. A 65, 043615 (2002).

[4] S. Gupta, K. Dieckmann, Z. Hadzibabic, and D. E. Pritchard, Phys. Rev. Lett. 89, 140401 (2002).

[5] I. Bloch, T. W. Hänsch, and T. Esslinger, Phys. Rev. Lett. 82, 3008 (1999).

[6] W. Ketterle, Rev. Mod. Phys. 74, 1131 (2002).

[7] V. M. Pérez-Garcia, H. Michinel, and H. Herrero, Phys. Rev. A 57, 3837 (1998).

[8] F. Dalafovo et al., Rev. Mod. Phys. 71, 463 (1999).

[9] S. Burger et al., Phys. Rev. A 65, 043611 (2002); L. D. Carr et al., ibid. 62, 063610 (2000); L. D. Carr and Y. Castin, ibid. 66, 063602 (2002); L. D. Carr, M. A. Leung, and W. P. Reinhardt, J. Phys. B 33, 3983 (2000).

[10] Z. X. Liang, Z. D. Zhang, and W. M. Liu, Phys. Rev. Lett. 94, 050402 (2005).

[11] V. V. Konotop and P. Pacciani, Phys. Rev. Lett. 94, 240405 (2005).

[12] F. S. Cataliotti et al., Science 293, 843 (2001).

[13] L. Khaykovich et al., Science 296, 1290 (2002).

[14] K. E. Strecker et al., Nature (London) 417, 150 (2002); U. Al Khawaja et al., Phys. Rev. Lett. 89, 200404 (2002).
[15] S. L. Cornish, S. T. Thompson, and C. E. Wieman, e-print cond-mat/0601664.

[16] J. M. Vogels et al., Phys. Rev. A 56, R1067 (1997).

[17] S. Inouye et al., Nature (London) 392, 151 (1998).

[18] J. L. Roberts et al., Phys. Rev. Lett. 85, 728 (2000).

[19] J. Janis, M. Banks, and N. P. Bigelow, Phys. Rev. A 71, 013422 (2005).

[20] J. D. Moores, Opt. Lett. 21, 555 (1996).

[21] V. I. Kruglov, A. C. Peacock, and J. D. Harvey, Phys. Rev. Lett. 90, 113902 (2003).

[22] V. N. Serkin and A. Hasegawa, IEEE J. Sel. Top. Quantum Electron. 8, 418 (2002); V. N. Serkin, A. Hasegawa, and T. L. Belyaeva, Phys. Rev. Lett. 92, 199401 (2004).

[23] T. S. Raju, C. N. Kumar, and P. K. Panigrahi, J. Phys. A 38, L271 (2005); T. S. Raju, P. K. Panigrahi, and K. Porsezian, Phys. Rev. E 71, 026608 (2005).

[24] V. E. Zhakarov and A. B. Shabat, Zh. Eksp. Teor. Fiz. 61, 118 (1972) [Sov. Phys. JETP 34, 62 (1972)].

[25] L. Salasnich, A. Parola, and L. Reatto, Phys. Rev. A 65 , 043614 (2002).

[26] H. Hancock, Theory of Elliptic Functions (Dover, New York, 1958); Handbook of Mathematical Functions, Natl. Bur. Stand. Appl. Math. Ser. No. 55, edited by M. Abramowitz and I. Stegun (U.S. GPO, Washington, DC, 1964).

[27] S. Kumar and A. Hasegawa Opt. Lett. 22, 372 (1997); V. N. Serkin and A. Hasegawa, Phys. Rev. Lett. 85, 4502 (2002). 